\title{
Recent Updates in Inflammatory Ocular Diseases
}

\author{
An Expert Interview with Bennie H Jeng \\ Department of Ophthalmology and Visual Sciences, University of Maryland School of Medicine, Baltimore, MD, US
}

DOI: https://doi.org/10.17925/USOR.2018.11.1.19

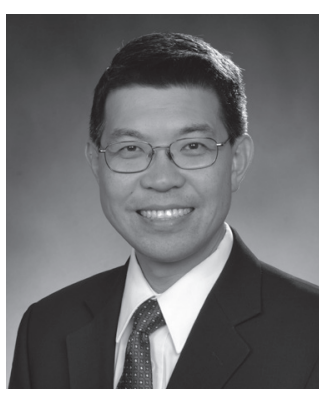

\begin{abstract}
Bennie H Jeng
Bennie H Jeng is Professor and Chair of the Department of Ophthalmology and Visual Sciences at the University of Maryland School of Medicine. He earned his bachelor's degree summa cum laude from Washington University and his medical doctorate from the University of Pennsylvania School of Medicine. He then completed his ophthalmology residency and chief residency at the Cole Eye Institute of the Cleveland Clinic, which was followed by a fellowship in cornea and external diseases at the Francis I. Proctor Foundation/University of California San Francisco (UCSF) in 2003. He then returned to the Cole Eye Institute to serve on the faculty, during which time he was the recipient of a Research Career Development Award from the National Institutes of Health and also earned his Master degree in Clinical Investigation. He subsequently returned to Proctor/UCSF as an Associate Professor and then Full Professor, where he served as co-director of the UCSF cornea service, Director of the Proctor/UCSF Cornea Fellowship program, and as Chief of Ophthalmology at the San Francisco General Hospital. He was a Research Project Grant-funded researcher at UCSF, and he assumed his current position at the University of Maryland in August, 2013. Dr Jeng is actively involved in the leadership of many of our ophthalmologic societies, including as a member of the Board of Directors of the Cornea Society, and the Contact Lens Association of Ophthalmologists. He also serves on several committees of the American Academy of Ophthalmology, including the Annual Meeting Program Committee, as well as The Council. He is also actively involved with the American Board of Ophthalmology. Dr Jeng is a past member of the editorial board of JAMA Ophthalmology, and he currently serves as an associate editor for three journals: Ophthalmology, Cornea, Eye and Contact Lens.
\end{abstract}

\section{Keywords}

Inflammatory ocular disease, MMP-9, tear osmolarity, lactoferrin, Sjögren's syndrome, Stevens-Johnson syndrome, allergic conjunctivitis

Disclosure: Bennie $\mathrm{H}$ Jeng has nothing to disclose in relation to this article.

Review Process: This is an expert interview with an Editorial Board member and as such did not undergo the journal's standard peer review procedure.

Authorship: All named authors meet the International Committee of Medical Journal Editors (ICMJE) criteria for authorship of this manuscript, take responsibility for the integrity of the work as a whole, and have given final approval to the version to be published.

open Access: This article is published under the Creative commons Attribution Noncommercial License, which permits any noncommercial use, distribution, adaptation, and reproduction provided the original author(s) and source are given appropriate credit. (C) The Authors 2018.

Received: March 19, 2018

Published Online: March 26, 2018

Citation: US Ophthalmic Review, 2018;11(1):19-20

Corresponding Author: Bennie $\mathrm{H}$ Jeng, Department of Ophthalmology and Visual Sciences, 419 West Redwood St, Suite 470, Baltimore, MD 21201, US. E: bjeng@som.umaryland.edu

Support: No funding was received in

the publication of this article. nflammatory ocular diseases cover a wide spectrum of conditions and are a growing public health concern. Dry eye disease is one of the most common conditions seen by eye care practitioners. Allergic conjunctivitis is also a highly prevalent condition, especially during hay fever season. In addition to these well characterized disorders, less-common inflammatory conditions such as Sjögren's syndrome and Stevens-Johnson syndrome also affect the eyes. Early diagnosis of these conditions is essential to optimize outcomes. In an expert interview, Bennie Jeng, Professor and Chair of the Department of Ophthalmology and Visual Sciences at the University of Maryland School of Medicine, discusses some of the latest advances in the diagnosis and treatment of inflammatory eye disorders.

\section{Q. What are the benefits and limitations of the MMP-9 test for dry eye?}

This point-of-care test detects the presence of matrix metalloproteinase 9 (MMP-9), an inflammatory marker that has been shown to be associated with dry eyes. Studies have demonstrated good sensitivity and excellent specificity. This test is easy and quick to do in the office, but it cannot quantitate the levels of MMP-9 to allow for tracking of improvement in dry eyes. As such, this test could be a useful adjunct when the diagnosis is in question.

\section{Q. What other new point-of-care tests appear promising in dry eye?} Other point-of-care tests include tear osmolarity and lactoferrin. Like the MMP-9 test, these other tests are also quick to do in the office, but are best used as adjuncts to careful examination of the ocular surface for staining and tear break up times. Osmolarity levels can be used to track improvement over time, allowing demonstration to the patients of an objective metric. 


\section{Q. Could you tell us a little about the Sjö kit for early detection of Sjögren's syndrome?}

The Sjö kit has been introduced as a method for early detection of Sjögren's syndrome. In addition to four traditional biomarkers, this kit also tests for three novel proprietary markers. With these new markers, the hope is that a diagnosis can be made sooner, thus allowing for earlier institution of therapy.

\section{Q. What have been the most exciting recent developments in the diagnosis and treatment of allergic conjunctivitis?}

There have been a number of newer therapies introduced that hold excellent promise for treating allergic conjunctivitis including sublingual immunotherapy (SLIT) and topical cetirizine. In addition, there are four new Food and Drug Administration (FDA)-approved products: Oralair ${ }^{\circledR}$ (sweet vernal, orchard, perennial rye, timothy and Kentucky bluegrass mixed pollens allergen extract), Grastek ${ }^{\circledR}$ (timothy grass pollen allergen extract), Ragwitek $^{\circledR}$ (short ragweed pollen allergen extract), and Odactra ${ }^{\mathrm{TM}}$ (house dust mite allergen extract).

\section{Q. What are the advantages of scleral lenses in patients with severe ocular disease such as Stevens-Johnson syndrome?}

Scleral lenses have recently become very popular for treating severe ocular surface diseases. This patient population does not respond adequately to standard medical treatments with tear supplements, and even autologous serum. Scleral lenses offer another medical option to avoid a surgical procedure such as amniotic membrane grafting or tarsorrhaphy. In addition, scleral lenses have the added advantage of improving quality of vision in addition to relief of symptoms. 